\title{
Metachronous Osteosarcoma: A Rare Case Report
}

Kow Ren Yi, Goh Kian Liang

Department of Orthopaedic Surgery, International Islamic University Malaysia.

Introduction: Osteosarcoma is a common primary sarcoma of the bone, constituting approximately $0.07 \%$ of all neoplasms. Rarely, some patients with osteosarcoma have multiple skeletal sites involvement, either synchronous or metachronous. Metachronous osteosarcoma is a rare form of osteosarcoma in which osteosarcomatous lesions occur distant from the primary osteosarcoma site more than 6 months after the initial treatment without pulmonary manifestation. Case Report: We present a case of metachronous osteosarcoma in a 16-year-old female who was initially treated for non-metastatic osteosarcoma of the left distal femur with neoadjuvant chemotherapy, wide excision, endoprosthesis and followed by adjuvant chemotherapy. Serial follow-up did not show evidence of distant metastasis. Nevertheless, she presented 3 years later with a progressively enlarging left proximal forearm mass and re-staging revealed an osteosarcoma of the same histological type. She underwent combination of chemo- and radio- therapy but subsequently succumbed to the disease due to lung metastasis. Conclusion: With the advent of diagnostic and therapeutic techniques, the 5-year survival rate of nonmetastatic osteosarcoma ranges around $60-70 \%$. Albeit it is rare, a metachronous osteosarcoma can present difficult challenges to the treating physician. 